\title{
ABSTRACTS OF PAPERS
}

\section{SUBMITTED FOR PRESENTATION TO THE SOCIETY}

The following papers have been submitted to the Secretary and the Associate Secretaries of the Society for presentation at meetings of the Society. They are numbered serially throughout this volume. Cross references to them in the reports of the meetings will give the number of this volume, the number of this issue, and the serial number of the abstract.

\section{Algebra And Theory of Numbers}

\section{A. A. Albert: The radical of a non-associative algebra.}

The multiplications of an algebra $A$ generate a corresponding associative polynomial algebra $T(A)$. Every ideal $B$ of $A$ determines a corresponding ideal $S$ of $T(A)$ and it is shown that $T(A-B)$ is equivalent to $T(A-S)$. If $H$ is the radical of $T(A)$ the set $A H$ is an ideal of $A$ and $A-A H$ is either a zero algebra $Z$, a semi-simple algebra $G$ (direct sum of simple algebras), or a direct $\operatorname{sum} Z \oplus G$. If $A-A H=Z$ the algebra $A$ is not homomorphic to a semi-simple algebra. For all other algebras the radical is defined to be the minimal ideal $N$ such that $A-N$ is semi-simple. Then $N=A H$ if $A-A H$ is semi-simple. Otherwise $Z=N-A H$, the quantities of $N$ are the quantities in the cosets which make up $Z$. If $A$ and $A_{1}$ are isotopic algebras with unity quantities, their radicals $N=A H$ and $N_{1}=A_{1} H_{1}$ are isotopes and the difference algebras $A-N$ and $A_{1}-N_{1}$ are isotopes. An example is given of an algebra $A$ with a unity quantity whose radical, with respect to our definition, is a field. (Received February 12,1942.)

142. B. H. Arnold: Rings of transformations of certain vector spaces. Preliminary report.

Eidelheit (Studia Mathematica, vol. 9 (1940), pp. 97-105) showed that the algebraic properties of the ring of all continuous linear transformations of a real Banach space into itself characterizes the Banach space up to an isomorphism. Mackey has extended this result to a more general class of spaces. In this paper the author shows what algebraic properties of the ring correspond to certain properties of the space, such as completeness, reflexivity, reflexivity of the completion, or being the conjugate of some space. Eidelheit's result is extended to the complex case and a set of conditions is given which are necessary and sufficient that an abstract ring be the ring of all continuous linear transformations of some space into itself. (Received March 3,1942.)

143. Emil Artin and Peter Scherk: On the sum of two sets of integers.

Let $A, B$, respectively, be sets of non-negative integers $a, b$. Let $C$ be the set of all integers of the form $a+b$. Let $A(x), B(x), C(x)$ denote the number of positive inintegers of the sets less than or equal to $x$. A few months ago, H. B. Mann succeeded in proving: If $0 \subset A$ and $0 \subset B$, and if $C(n)<n$, then $C(n) / n \geqq \min { }_{x=1,}, \ldots, n(A(x)$ $+B(x)) / x$. Applying and simplifying Mann's method, the authors have proved: If $C(n)<n$, then $C(n)-C(n-m)=A(m-1)+B(m-1)+Z_{m}(n)$, for a suitable $m \nsubseteq C$ 
with $0<m \leqq n ; Z_{m}(n)$ denotes the number of decompositions of $n$ of a certain type (hence $Z_{m}(n) \geqq 0$ ). If $0 \subset A, 0 \subset B$, this result contains various estimates of $C(n)$ including that by Mann. If $0 \subset A$ a result of $\mathrm{A}$. S. Besicovitch's follows (Journal of the London Mathematical Society, vol. 10 (1935), pp. 246-248). If $0 \nsubseteq A, 0 \nsubseteq B$, new estimates of $C(n)$ follow immediately. (Received March 14, 1942.)

144. S. P. Avann: A numerical condition for modularity of a finite lattice.

Let $U(a)$ be the number of elements other than $a$ which are covered by elements covering $a$, an element of a finite lattice $L$ in which the Jordan-Dedekind chain condition holds; let $V(a)$ be the number of elements other than $a$ which cover elements covered by $a$. Then a necessary and sufficient condition that $L$ be modular is that $U(a)=V(a)$ for every $a \in L$. Finiteness of $L$ is required for sufficiency. Hence $L$ may be tested for modularity by counting coverings or bonds in the Hasse diagram of $L$. (Received March 14, 1942.)

\section{S. P. Avann: On complete classes of projective quotients in a} modular lattice.

Let $L$ be a finite modular lattice in which $Q$ is a complete class: $\left[a_{1} / b_{1}, a_{2} / b_{2}, \cdots\right.$, $\left.a_{r} / b_{r}\right]$ of projective quotients defined by the equivalence relation of projectivity. Denote the set of numerators $a_{i}$ by $A$ and the set of denominators $b_{i}$ by $B$. Then the following conditions are equivalent: (1) $A$ and $B$ are transposed quotient sublattices of $L$, (2) the meet $a_{0}$ of the $a_{i}$ is in $A$ and to each $a_{i} \neq a_{0}$ belongs a unique denominator $b_{i}$ in $B,\left(2^{\prime}\right)$ the join $b$ of the $b_{i}$ is in $B$ and to each $b_{i} \neq b$ belongs a unique numerator $a_{i}$ in $A$, (3) $a_{0} \in A$ and $b \in B$, (4) uniqueness of denominators and numerators. If the quotient sublattices of $Q$ are complemented then (1), (5) $a_{0} \in A,\left(5^{\prime}\right) b \in B$, (6) uniqueness of denominators, $\left(6^{\prime}\right)$ uniqueness of numerators, aare equivalent conditions. (Received March 14, 1942.)

\section{Reinhold Baer: Radical ideals.}

An ideal is termed a radical ideal, if it is a two-sided nil ideal modulo which there do not exist nilpotent right-ideals different from 0 . Every ring possesses at least one radical ideal; and both the cross-cut and the sum of all the radical ideals are themselves radical ideals which are called the lower and the upper radical, respectively. It is possible that lower and upper radical are different and that neither of them is nilpotent. If every right ideal, not 0 , in the quotient ring modulo the lower radical contains a smallest right ideal different from 0 , then upper and lower radical are equal; and if this condition is satisfied by every quotient ring, then a finite or transfinite power of the radical ideal is 0 . As an application of this theory a generalization of a theorem of C. Hopkins is proved. (Received March 12, 1942.)

147. Richard Brauer and Hsio-Fu Tuan: Some remarks on simple groups of finite order. Preliminary report.

Using the theory of group characters, some lemmas on simple groups of finite order are obtained. For instance, a group $G$ of order $3 \cdot p^{a} \cdot q^{b}$ ( $p, q$ primes) can only be simple if $G$ has one of the orders 60 or 168. It can be shown that the list of simple groups (cf. L. E. Dickson, Linear Groups) is complete up to the order 10,000. (Received March 6, 1942.) 


\section{Leonard Carlitz: On the reciprocal of certain types of Hurwitz} series.

In the first part of this paper previous results (Duke Mathematical Journal, vol. 9 (1942) ) are extended to series $\sum A_{m} t^{p^{n m}} / F_{m}$, where the $A_{m}$ are polynomials of a more general type. In the second part of the paper the case $\sum A_{m} t^{m} / G_{m}$ is treated. (Received March 6, 1942.)

\section{R. F. Clippinger: Matrix products of matrix powers.}

If $A_{1}, A_{2}, \cdots, A_{m}$ are given matrices of constants, the closure of the set of products of arbitrary positive powers of the matrices $\bar{A}_{1}=\exp A_{1}, \bar{A}_{2}=\exp A_{2}, \cdots, \bar{A}_{m}=$ $\exp A_{m}$ is identical with the closure of the set of solutions for positive $t$ of the matrix differential equation: $d y / d t=Y\left(\rho_{1}(t) A t_{1}+\rho_{2}(t) A_{2}+\cdots+\rho_{m}(t) A_{m}\right), Y(0)=1$, where $\rho_{1}(t), \rho_{2}(t), \cdots, \rho_{m}(t)$ are arbitrary, non-negative, measurable, bounded functions of t. (Received March 19, 1942.)

\section{R. P. Dilworth: The partition lattice.}

In a previous paper (abstract 48-1-11) a dependence relation for elements of rank $k$ in a Birkhoff lattice was defined. In this paper it is shown that if this dependence relation is applied to the elements of rank two in a Boolean algebra, the closed subsets form a lattice isomorphic to the partition lattice. (Received March 9, 1942.)

151. F. A. Ficken: On Rosser's generalization of Euclid's algorithm. Preliminary report.

Let $X_{1}, \cdots, X_{n}$ be a set of vectors in an euclidean space. The set of independent vectors $Y_{1}, \cdots, Y_{m}$ is a greatest common factor (G.C.F.) of the $X$ 's if each $X_{j}$ $=\sum p_{j i} Y_{i}$ and each $Y_{k}=\sum q_{k i} X_{i}$, where the $p$ 's and $q$ 's are integers. In a minimal G.C.F., the lengths of the $Y^{\prime}$ 's satisfy certain minimal conditions. Full definitions and a number of important applications may be found in Duke Mathematical Journal, vol. 9 (1942), pp. 59-95. Rosser has given there an algorithm for finding G.C.F.'s and all minimal G.C.F.'s. By examining the termination of the algorithm, he establishes several properties of minimal G.C.F.'s. In the present paper, this examination is conducted by producing explicit lower bounds for $\left(\sum t_{i} X_{i}\right)^{2},(i=1, \cdots, n ; n=2,3,4,5)$. These bounds furnish simplified proofs for the bulk of the results and confirm certain of the conjectures stated in Rosser's paper. (Received March 6, 1942.)

\section{J. S. Frame: Double coset matrices and group characters.}

This paper extends to the characters of the irreducible components of an intransitive permutation group a theorem proved earlier by the author (this Bulletin, vol. 47 (1941), p. 459) for the degrees of the irreducible components of a transitive permutation group. To the element $\gamma$ of $G$ corresponds the $n \times n$ permutation matrix $R(\gamma)$ with the $n^{t} \times n^{t}$ transitive constituents $R^{t}(\gamma)$ which contain the irreducible representation $\Gamma_{i}$ just $\mu_{i}^{t}$ times. To each transitive constituent corresponds a subgroup $H^{t}$ of order $h^{t}$ leaving its first symbol fixed. The matrices $V$ permutable with $G$ break up into submatrices $V^{t s}$ linearly dependent on $\mu^{t s}$ double coset matrices $V_{\alpha}^{t s}$, each associated with a double coset $H_{\alpha}^{s t}=H^{s} \gamma_{\alpha} H^{t} / h_{\alpha}^{s t}$ formed from the given subgroups. With the structure coefficients $c_{\alpha \beta \eta}^{r s t}$ which appear in the product formula $H_{\alpha}^{r s} H_{\beta^{\prime}}^{s t} / h^{s}=$ $\sum_{\eta} c_{\alpha \beta \eta}^{\text {rot }} H_{\eta}^{r t}$, with the constants $k_{\eta \lambda}^{t}$ which tell how many conjugate elements from the class $C$ lie in the double coset $H_{\eta}^{t}$, and with a set of arbitrary parameters $a_{\lambda}$, a matrix 
$K^{s t}=\sum_{\lambda, \eta} c_{\alpha \beta \eta}^{t s t} k_{\eta \lambda}^{t} a_{\lambda}$ is formed. Its determinant $\left|K^{s t}\right|$ will factor into linear factors $\chi_{i}=\chi_{i}\left(\sum a_{\lambda} C_{\lambda}\right)$ which give the characters of the irreducible components $\Gamma_{i}$ common to two constituents $R^{t}(\gamma)$ and $R^{s}(\gamma)$. The theorem is: $\left(n^{t}\right) \mu^{s t}\left|K^{s t}\right|=\mathrm{P}_{s t} \overline{\mathrm{P}}_{s t} \prod_{i}\left(\chi_{i}\right) \mu_{i}$, where $\mu_{i}=\mu_{i}^{t} \mu_{i}^{i}, \mu^{s t}=\sum \mu_{i}$, and $\mathbf{P}_{s t}$ is an algebraic integer. An illustrative example is given. (Received March 5, 1942.)

\section{R. D. James: On Euler's conjecture.}

It was conjectured by Euler that every integer $N \equiv 2(\bmod 4), N \geqq 10$, could be represented as a sum of two primes each congruent to $1(\bmod 4)$. However even the weaker statement that every such integer is a sum of two integers which have all their prime factors congruent to $1(\bmod 4)$ has not yet been proved. In a previous paper (Transactions of this Society, vol. 43 (1938) pp. 296-302) the author proved that every sufficiently large integer $N \equiv 2(\bmod 4)$ is a sum of two integers which have all except possibly two of their prime factors congruent to $1(\bmod 4)$. This result is improved in the present paper as indicated in the following theorem: Every sufficiently large integer $N \equiv 2(\bmod 4)$ is a sum of two integers which either have at most six prime factors all congruent to $1(\bmod 4)$ or else have exactly two prime factors congruent to $3(\bmod 4)$ and at most three prime factors congruent to $1(\bmod 4)$. The method of proof is a general one and applies to any infinite sequence of primes for which $\sum p^{-1} \log p=\mu \log x+O(1)$, where $\mu$ is any positive number. (Received March 20, 1942.)

\section{R. E. Johnson: On structures of infinite modules.}

Let $Q$ be a principal ideal ring containing the field $P$, and $\Xi$ be a $P$-module which has $Q$ as an operator domain. It is assumed that $\Xi$ has a countable $P$-basis. Then the problem studied is under what conditions there exist elements $\xi_{1}, \xi_{2}, \ldots$ in $\Xi$ such that $\Xi=Q \xi_{1}+Q \xi_{2}+\cdots$, this last being a supplementary sum. Necessary and sufficient conditions are found for this to be the case. If $Q /(m), m$ not a unit of $Q$, is taken as the operator domain of $\Xi$, then there always exist elements $\xi_{1}, \xi_{2}, \cdots$, of $\Xi$ such that $\Xi=Q /(m) \xi_{1}+Q /(m) \xi_{2}+\cdots$. Now let $\mathfrak{M}_{\omega}$ be the matrix algebra of order type $\omega$ over $P$ with finitely nonzero columns, and $\Xi$ be the $P$-module composed of the columns of these matrices. Then an element $A$ of $\mathfrak{M}_{\omega}$ can be transformed into a reduced form in case $\Xi=P[A] \xi_{1}+P[A] \xi_{2}+\cdots$. (Received March 18, 1942.)

\section{H. F. S. Jonah: Congruences connected with the solution of a certain Diophantine equation.}

The paper begins with a function and functional equation used by Maier (Mathematische Annalen, vol. 104 (1931), pp. 745-769) and generalizes both. Then by means of the calculus of residues the author obtains a quadratic functional equation of a function closely related to the $h_{n}^{(k, l)}(x)$ of Vandiver (Annals of Mathematics, (2), vol. 27 (1926), pp. 171-176). Using this functional equation it is possible to transform the general congruences used by Vandiver in his work on Fermat's last theorem. (Received March 18, 1942.)

\section{Joseph Lehner: On certain irrational modular equations.}

The author obtains by simple group-theoretic considerations the modular equation connecting $\eta(\tau) / \eta(\tau / p)$ and $\eta(p \tau) / \eta(\tau)$, where $\eta(\tau)=\exp (\pi i \tau / 12) \cdot \prod_{1}^{\infty}(1-\exp$ $(2 \pi i n \tau)$ ) and $p=5,7,13$. These are the only three primes $p$ for which an equation of degree $p$ exists, this fact being connected with the genus of the subgroup 
$\Gamma_{0}(p)$ of the full modular group. The equations for $p=5,7$ were obtained by Watson (Crelle, 1937) by more complicated methods; they are important in the theory of Ramanujan's congruences concerning the unrestricted partition function, $p(n)$. (Received February 21, 1942.)

157. F. A. Lewis: Generators of permutation groups simply isomorphic with $\operatorname{LF}\left(2, p^{n}\right)$.

Generators of any permutation group of the class are obtained by a slight extension of an argument used in a recent paper (this Bulletin, vol. 47 (1941), p. 631). (Received February 20, 1942.)

\section{Knox Millsaps: Abstract polynomials in non-abelian groups.}

After an abstract calculus of finite differences is defined, functional definitions of a monomial and a polynomial for elements of the central subgroup or elements of the group as increments are given. The theorem on the homogeneity of a polynomial is proved for central and arbitrary differences; for central differences the difference being a function of the increment alone implies the difference is a monomial; the independence of the central difference of polynomials and the unique decomposition for the abelian valued case are made to depend on the product of a Vandermonde determinant and a finite product of binomial coefficients; and these last results can be extended to the non-abelian valued case for certain free groups. The theory is essentially a generalization of the work of Van der Lijn on abstract polynomials in abelian groups. (Received March 4, 1942.)

\section{Ivan Niven: On matric polynomials.}

Let $B$ be a matrix with elements from any field, and let $P$ be a scalar polynomial in $B$ with coefficients from the field. Then the index of $P$ cannot exceed the index of $B$. Furthermore, $B$ is expressible as a polynomial in $P$ if and only if the index of $P$ equals the index of $B$. A new method is offered for finding those solutions $X$ of $X^{m}=A$ which are polynomials in $A$, the elements of $A$ being from an algebraically closed field with characteristic 0 (cf. M. H. Ingraham, Rational methods in matrix equations, this Bulletin, vol. 47 (1941), p. 64, Theorem 3). If $A$ is non-derogatory every solution of $X^{m}=A$ is a polynomial in $A$, and the equation has no solutions if and only if 0 is a repeated root of the minimal equation of $A$. (Received March 17, 1942.)

\section{Rufus Oldenburger: Expansions of quadratic forms.}

A quadratic form $Q$ is ordinarily given as a sum $a_{i j} x_{i} x_{j}$ of power products of the variables. Although this is a sum of products of linear forms, this is not in general the most economical way of writing $Q$ as such a sum. The minimum $\tau$ such that $Q$ is a sum of $\tau$ products of linear forms is $r-\sigma$, where $r$ is the rank of $Q$, and $\sigma$ is the index of $Q$ introduced elsewhere by the author. This result is valid for fields with characteristic not 2. (Received February 12, 1942.)

\section{Rufus Oldenburger: Repeated linear factors of forms.}

With a form $F$ of degree $n$ there are associated 2-way displays, $A_{0}, A_{1}, \cdots, A_{n}$ of the symmetric matrix $A$ of coefficients of $F$, used by W. Mayer, Thrall and Chandler, F. L. Hitchcock, and others. The form $F$ has a linear factor $L$ repeated $r$ times if and only if $L^{n-r+1}$ is a covariant directly constructible from $A_{n-r+1}$. If $n$ is even the coefficients in $L^{n-r+1}$ can be expressed simply as higher-dimensional determinants. (Received February 20, 1942.) 
162. Rufus Oldenburger: The index of a quadratic form for an arbitary field.

The classical index of a quadratic from $Q$ is defined in terms of the order of elements in the real field. In the present paper a definition of the index of $Q$ is given which is valid for any field, and which reduces in the real case to the index of $Q$ or $-Q$. It is proved that a form $Q$ with rank $r$ and index $\sigma$ has a splitting $G+H$, where $G$ has index $\sigma$ and rank $2 \sigma$, whereas $H$ has index 0 and rank $r-2 \sigma$. A second form $Q^{\prime}$ with a corresponding splitting $G^{\prime}+H^{\prime}$ is equivalent under a nonsingular linear transformation to $Q$ if and only if the rank and index of $Q$ equal the rank and index of $Q^{\prime}$, and $H$ is equivalent to $H^{\prime}$. A form $H$ has index 0 if and only if $H$ is a nonzero form. Thus the problem of the equivalence of quadratic forms reduces to that of the equivalence of nonzero forms. Such forms have been studied by Siegal, Weyl, Dickson, Legendre, and many others. (Received February 13, 1942.)

\section{M. A. Sadowsky: Approximation by rational sequences.}

A certain graphical principle is employed to select the best approximations to a real number. It is shown that this process leads to the consecutive convergents of the continued fraction development. (Received March 20, 1942.)

\section{R. D. Schafer: Alternative algebras over an arbitrary field.}

The results of M. Zorn concerning simple alternative algebras are incomplete for algebras over modular fields of characteristics two or three. This paper presents a unified treatment of such algebras over an arbitrary field. Define a quaternion algebra $Q$ over $F$ as in A. A. Albert's Structure of Algebras, American Mathematical Society Colloquium Publications, vol. 24, 1939, p. 145. Then an alternative, but not associative, algebra $A$ over $F$ is central simple (simple for all scalar extensions) if and only if $A$ is a Cayley-Dickson algebra $A=Q+g Q$, in which multiplication is defined by $\left(q_{1}+g q_{2}\right)\left(q_{3}+g q_{4}\right)=\left(q_{1} q_{3}+\delta q_{4} \cdot q_{2} S\right)+g\left(q_{1} S \cdot q_{4}+q_{3} q_{2}\right)$, where $g^{2}=\delta \neq 0$ in $F$ and $S$ is the involution $z \leftrightarrow z S=t(z)-z$, the principal function of $A$ being $z^{2}-t(z) \cdot z+n(z)$. Moreover $A$ is a central division algebra if and only if $Q$ is a division algebra and there exist no $\lambda, \mu, \rho, \sigma$ in $F$ such that $\delta=\lambda^{2}+\lambda \mu-\beta \mu^{2}-\gamma \rho^{2}-\gamma \rho \sigma+\beta \gamma \sigma^{2}$. (Received March 20, 1942.)

165. M. F. Smiley and W. R. Transue: Applications of transitivities of betweenness in lattic theory.

This paper is an expansion of our note Metric lattices as singular metric spaces (this Bulletin, abstract 48-1-20) which was to appear in this Bulletin. The properties of betweenness studied in the original note are generalized and applied to the characterization of modular lattices and of arbitrary lattices. The proofs of the extensions of our original results are based in part on certain of the results of the paper Transitivities of betweenness by Everett Pitcher and M. F. Smiley (forthcoming in the Transactions of this Society). (Received February 27, 1942.)

\section{R. M. Thrall: On the decomposition of modular tensors.}

Let $k$ be any field, and denote by $A$ any element of the full linear group $G(n, k)$ of $n$-rowed $k$-matrices. The mapping $A \rightarrow \pi^{m}(A)=A \times \cdots \times A$ (Kronecker direct product with $m$ factors $A$ ) is a representation of $G(n, k)$ as linear transformations on the space $V_{m}$ of all tensors of rank $m$. For $k$ of characteristic zero the theory of the decomposition of $V_{m}$ into invariant subspaces is classical. A key tool of the develop- 
ment of this theory is use of the relationship between $V_{m}$ and the symmetric group of degree $m$. For $k$ of characteristic $p$ the classical theory is valid only for $m<p$. In the present paper the decomposition of $V_{m}$ is obtained for $p \leqq m<2 p$. Further progress on the decomposition problem will likely have to await the development of the theory of modular representations of the symmetric groups of degree $m \geqq 2 p$; the measure of difficulty here being the power of $p$ which divides $m$ !. (Received March 26, 1942.)

\section{Bernard Vinograde: Split rings and their representation theory.}

Suppose a ring $R$, with minimum condition on left ideals, can be split into a direct sum, $R=R^{*}+N$, of a semi-simple ring and the radical. This splitting property of $R$ is equivalent to the existence of a certain set of noncommutative fields in $R$. Let $R$ have a unit, and let $V$ be a commutative group possessing $R$ in its left operator domain and a finite composition series with respect to $R$. Then $V$ is a direct sum of modules, each over one of the noncommutative fields. Thus $V$ gives rise to a composite representation module for its homomorphism ring. In the case $V=R$ the resulting representation of $R$ displays the essential structure of $R^{*}$ and $N$, and may be considered a natural extension of the Wedderburn theorem for simple rings to the class of split rings with unit and finite composition series. (Received March 20, 1942.)

168. R. W. Wagner: Expressibility relations for bilinear operations. Preliminary report.

If $x y$ is a multiplication operation for a vector space over a field, an expression like $(a((x b)(c y))) d$ is also a multiplication for the vector space. The operation $x^{*} y$ is called simply expressible in the operation $x y$ if $x^{*} y$ is a finite iteration of the original operation. The operation $x^{*} y$ is called strongly expressible if it is a linear combination of weakly expressible operations. Necessary and sufficient conditions are found for both kinds of expressibility. These conditions involve the enveloping algebra of the algebra formed by the original multiplication. Mutual expressibility, both simple and strong, is an equivalence relation and differs from isotopy. Reducibility is a property common to mutually expressible algebras. Associativity, commutativity, and the existence of nilpotents are properties which need not be preserved. (Received March 18, 1942.)

\section{Analysis}

169. Dorothy L. Bernstein and S. M. Ulam: On the problem of completely additive measure in classes of sets with a general equivalence relation.

The problem of finding a necessary and sufficient condition for the existence of a finitely additive measure in a class of sets, with the property that equivalent sets have equal measure, has been solved by Tarski (Fundamenta Mathematicae, vol. 31 (1938), pp. 47-66). This paper considers the existence of a completely additive measure in a class $K$ which is a Borel field over a given sequence $\left\{A_{n}\right\}$ of sets and which has the property that $m(X)=m(Y)$ if $X$ is equivalent to $Y$. Equivalence is defined in the most general sense: Given a division of the sets of $K$ into disjoint classes $A_{1}, A_{2}, A_{3}, \cdots$, sets $X$ and $Y$ are called equivalent if they belong to the same class $A_{\alpha}$. It should be noted that even if no requirement for equivalence is made, it is not always possible to find a measure in a Borel field over a given sequence of sets. A necessary and suffi- 